\title{
gु \\ Subband engineering even-denominator quantum Hall states
}

\author{
V. W. Scarola, ${ }^{1}$ C. May, ${ }^{2}$ M. R. Peterson, ${ }^{3}$ and M. Troyer $^{2}$ \\ ${ }^{1}$ Department of Physics, Virginia Tech, Blacksburg, Virginia 24061, USA \\ ${ }^{2}$ Theoretische Physik, ETH Zurich, 8093 Zurich, Switzerland \\ ${ }^{3}$ Condensed Matter Theory Center, Department of Physics, University of Maryland, College Park, Maryland 20742, USA
}

(Received 19 August 2010; published 9 September 2010)

\begin{abstract}
Proposed even-denominator fractional quantum Hall effect (FQHE) states suggest the possibility of excitations with non-Abelian braid statistics. Recent experiments on wide square quantum wells indicate evendenominator FQHE even under electrostatic tilt. We theoretically analyze these structures and develop a procedure to accurately test proposed quantum Hall wave functions. We find that tilted wells favor partial subband polarization to yield Abelian even-denominator states. Our results show that tilting quantum wells effectively engineers different interaction potentials allowing exploration of a wide variety of evendenominator states.
\end{abstract}

DOI: 10.1103/PhysRevB.82.121304

PACS number(s): 73.43.-f, 71.10.Pm

Experimental evidence for more than 70 odd-denominator (FQHE) states ${ }^{1}$ demonstrates the ubiquity of incompressible quantum Hall electron liquids in high mobility semiconductor quantum wells. The Laughlin wave functions ${ }^{2}$ describe a few of these states, however, the composite fermion (CF) theory includes Laughlin states into a comprehensive framework that captures the essential physics of the observed lowest Landau-level (LLL) FQHE states., ${ }^{3,4}$

Observations of rare even-denominator FQHE states, exceptions to the odd-denominator rule, occur in multicomponent systems ${ }^{5}$ and at half filling of the second LL, ${ }^{6}$ i.e., filling factor $5 / 2$. Spin, layer, or subband degrees of freedom allow combinations of single-component odd-denominator states to yield even-denominator fractions. ${ }^{7}$ But at half filling of the second LL theoretical analyses ${ }^{8}$ suggest that the Coulomb interaction happens to favor the formation of a singlecomponent paired CF state, the Pfaffian, ${ }^{9}$ a profoundly distinct state.

Proposed FQHE states possess topologically nontrivial excitations. The common $\mathrm{CF}$ states carry excitations that obey Abelian anyonic braid statistics. Braiding two Abelian excitations changes the overall wave function by a phase., ${ }^{4,10}$ In contrast, excitations above the $\mathrm{CF}$ paired Pfaffian state obey non-Abelian braid statistics; a braid operates on the overall wave function by a matrix. Non-Abelian excitations have potential application in topological quantum information processing making observation of non-Abelian excitations a key goal. ${ }^{11}$

Experimental parameters (width and density) in narrow quantum-well samples allow only limited tunability. Recent diagonalization studies ${ }^{12}$ indicate that in a single-layer system the Pfaffian state is likely (unlikely) to be the ground state for $\nu=5 / 2(1 / 2)$ within the simple Coulomb interaction model. By chance, including finite thickness of realistic quantum wells marginally favors the formation of the Pfaffian state in the second LL. ${ }^{13}$ A route toward the exploration of putative non-Abelian phases and their phase boundaries requires construction of tunable high mobility quantum wells designed to favor these fragile states by engineering the effective electron-electron interaction.

Recent experiments with wide square quantum wells demonstrate even-denominator LLL FQHE in a regime with a surprisingly strong interlayer tunneling ${ }^{14,15}$ suggesting that explanations in terms of the usual Abelian multicomponent states need to be revisited. Do these observations suggest that the new samples effectively tailor the interaction to favor a LLL Pfaffian state? ? $^{15} 16$ The competition between the (non-Abelian) Pfaffian and (Abelian) multicomponent states remains a subtle issue. ${ }^{16,17}$ Furthermore, it is currently unknown whether these or similar samples will favor a second LL Pfaffian or entirely new FQHE states.

In this Rapid Communication, we show that recent experiments observing even-denominator FQHE (Refs. 14 and 15) in wide quantum wells occur in a regime that can favor partial subband occupancy and therefore offer the ability to engineer a wide array of even-denominator FQHE states. We develop a protocol that combines a modified high-field localdensity approximation (LDA) of quantum-well subbands with $\mathrm{FQHE}$ wave functions in the plane of the quantum well. Focusing on the experimental parameters of Ref. 15 we argue that evidence of even-denominator FQHE in tilted samples arises from partially subband polarized Abelian FQHE states ${ }^{18}$ and, in turn, demonstrate the remarkable ability to tune among subband balanced and imbalanced FQHE states. Our protocol also applies to the second LL, where subband engineering can be used to tune and explore second LL even-denominator states. ${ }^{17}$

We begin with a model of square quantum wells of wide width, where at most two subbands are populated. We construct a procedure to energetically minimize the following three-dimensional model in a strong perpendicular magnetic field, $B$ :

$$
H^{3 \mathrm{D}}=K+V_{\mathrm{W}}+V+H_{\mathrm{b}},
$$

where $K$ is the electron kinetic energy, $V_{\mathrm{W}}$ is the quantumwell confinement potential, $V=\sum_{i \neq j} e^{2} /\left(2 \epsilon\left|\mathbf{r}_{i}-\mathbf{r}_{j}\right|\right)$ is the three-dimensional Coulomb interaction, $\epsilon$ is the dielectric constant, and $H_{\mathrm{b}}$ accounts for the energy of a plane of rigid positive background charges. In what follows we consider a tilted square quantum well to model a specific set of experimental parameters ${ }^{15}$ as an application of our procedure: 
$V_{\mathrm{W}}\left(z_{\perp}\right)=\alpha z_{\perp}+V_{0} \theta\left(\left|z_{\perp}\right|-w / 2\right)$. The extra potential, $\alpha z_{\perp}$, tilts the well. We choose $V_{0}=270 \mathrm{meV}$ and a well width of $w=55 \mathrm{~nm}$.

To determine the tilt parameter $\alpha$ we first consider the $B=0$ limit. To solve for $\alpha$ we assume a Fermi sea in the plane and apply usual LDA methods. ${ }^{19}$ Here we assume that the electron spins are unpolarized and use the HedinLundqvist exchange-correlation energy ${ }^{20}$ at a planar density of $\rho=1.72 \times 10^{11} \mathrm{~cm}^{-2}$. We find that the density imbalance $\left(7.8 \times 10^{10} \mathrm{~cm}^{-2}\right)$ and subband splitting measured in Ref. 15 $\left(\Delta_{01}^{B=0}=41 \mathrm{~K}\right)$ are reproduced with $\alpha=0.3 \mathrm{meV} / \mathrm{nm}$.

We use this value of $\alpha$ to connect to the high-field experiments of Ref. 15 and now consider the high field, LLL limit of $H^{3 \mathrm{D}}$. The planar and perpendicular coordinates separate and we assume that in the $x y$ plane the electrons are in the LLL with a quenched kinetic energy and basis states given by: $\quad \phi_{m}\left(z / l_{0}\right)=\left(z / l_{0}\right)^{m} \exp \left(-|z|^{2} / 4 l_{0}^{2}\right) /\left(l_{0} \sqrt{2 \pi 2^{m} m !}\right)$, where $z=x-i y$ and $l_{0}=\sqrt{\hbar c / e B}$ is the magnetic length. We also assume that the real spins are fully polarized (the experiments at total filling $\nu=1 / 2$ in Ref. 15 are performed for $B \approx 14 \mathrm{~T})$. We then use LDA with a spin-polarized exchange-correlation energy ${ }^{21}$ to model the electron wave function perpendicular to the plane.

In wide wells the subband polarization, $\gamma=\left(N_{0}-N_{1}\right) / N$, is a key unknown connecting the energetics of the plane to the energetics arising from perpendicular coordinates. Here $N_{0}$ $\left(N_{1}\right)$ denotes the number of electrons in the lowest (first) subband with $N=N_{0}+N_{1}$. At zero field $\gamma$ is determined by the energetics of the $x y$-plane Fermi surface ${ }^{19}$ but this is not obviously accurate in wide quantum wells in the LLL.

To determine the ground state we minimize the total energy per particle, $E_{\gamma}^{\text {total }}=E_{\gamma}^{\perp}+E_{\gamma}^{x y}$, where $E_{\gamma}^{\perp}=\left(N_{0} E_{0}+N_{1} E_{1}\right) / N$, the weighted sum of both subband energies, arises from the quantum-well confinement and $E_{\gamma}^{x y}$ is the correlation energy due to the LLL planar Coulomb interaction. We calculate $E^{\text {total }}$ with the following procedure: for each value of $\gamma$ we use LDA to compute the confinement energy, $E_{\gamma}^{\perp}=\Delta_{\mu}^{\gamma} / 2-\gamma \Delta_{01}^{\gamma} / 2$, rewritten here in terms of the $\gamma$-dependent subband energy difference, $\Delta_{01}^{\gamma}$, and an effective chemical potential, $\Delta_{\mu}^{\gamma}$. The LDA also yields $\xi_{\sigma}$, where $\xi_{0}$ and $\xi_{1}$ denote the lowest and first subband wave functions, respectively. The LDA output is used to construct an effective two-dimensional model of spin-polarized LLL fermions, $H_{\gamma}^{\mathrm{eff}}=H_{\gamma}^{\perp}+H_{\gamma}^{x y}$, one at each $\gamma$. Finally, we compute $E^{x y}$ with two-component variational FQHE wave functions in the subband basis at fixed $\gamma$ thus allowing global minimization of $E_{\gamma}^{\text {total }}$ in the space of competitive FQHE wave functions. Our procedure results in a set of models, $H_{\gamma}^{\text {eff }}$, that are very sensitive to the quantum-well parameters showing that experiments can tune through a large set of multicomponent interactions.

To model the perpendicular degrees of freedom we introduce an effective model that captures the subband energetics,

$$
H_{\gamma}^{\perp}=\sum_{m}\left[\frac{\Delta_{01}^{\gamma}}{2}\left(n_{m, 1}-n_{m, 0}\right)-\frac{\Delta_{\mu}^{\gamma}}{2}\left(n_{m, 1}+n_{m, 0}\right)\right],
$$

where $n_{m, \sigma}=c_{m \sigma}^{\dagger} c_{m \sigma}$ and $c_{m \sigma}^{\dagger}$ creates an electron in the state $\xi_{\sigma} \phi_{m} . H^{\perp}$ parametrizes interacting electrons at fixed $\gamma$ in the

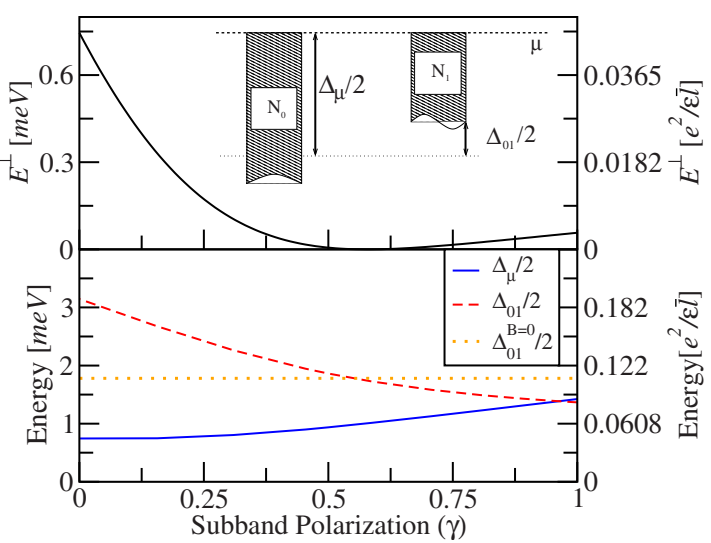

FIG. 1. (Color online) Top: confinement energy plotted as function of subband polarization for parameters corresponding to tilted wide well samples of Ref. 15. The right axis shows Coulomb units in terms of the fixed magnetic length, $\bar{l}$, obtained for $\nu=1 / 2$ and $\rho=1.72 \times 10^{11} \mathrm{~cm}^{-2}$. Bottom: the same but for the subband energy difference (dashed) and the chemical potential offset (solid). The dotted line plots the $B=0$ subband energy difference. Inset: schematic showing partial subband occupancy captured by an effective chemical potential.

perpendicular direction as noninteracting fermions with an effective chemical potential. The first term is the subband splitting commonly used in models of very wide wells but the second term is added to capture the energetics of partial subband occupation in wide wells. The second term allows calculation of the total energy when $\Delta_{\mu}^{\gamma}$ varies with $\gamma$. The inset of Fig. 1 shows that $\Delta_{\mu}>0$ favors partial subband occupancy. We obtain the very wide-well limit for $\Delta_{\mu} \gg \Delta_{01}$ and the narrow-well limit with $\Delta_{\mu} \ll \Delta_{01}$.

In the top panel of Fig. 1 we see that the lowest energy is obtained for subband polarization $\gamma \approx 0.59$. The large upturn near $\gamma=0$ arises because the well tilt strongly penalizes occupancy of the second subband. The upturn near $\gamma=1$ arises from the Coulomb cost of putting all charges in the lowest subband. The bottom panel of Fig. 1 plots $\Delta_{\mu}^{\gamma}$ and $\Delta_{01}^{\gamma}$ versus $\gamma$ to show that they vary in comparison to the subband splitting obtained from the zero-field calculation, $\Delta_{01}^{B=0}$. The nonlinearity demonstrates a Stoner-type dependence on the subband-pseudospin occupancy that arises from the competition between the kinetic energy, well tilt, and Coulomb interaction along the direction perpendicular to the plane.

We now use output from our $\gamma$-dependent LDA calculation, $\xi_{\sigma}$, to construct an effective interaction within the plane. The LLL interaction contains matrix elements of the form: $V_{m_{1} m_{2} m_{3} m_{4}}^{\sigma_{1} \sigma_{2} \sigma_{3} \sigma_{4}}=\left\langle m_{1}, m_{2}\left|V_{\{\sigma\}}(r)\right| m_{3}, m_{4}\right\rangle$. The effective planar interaction is given by: $V_{\{\sigma\}}(r)=\left\langle\xi_{\sigma_{1}}, \xi_{\sigma_{2}}|V| \xi_{\sigma_{3}}, \xi_{\sigma_{4}}\right\rangle$. The inset of Fig. 2 plots the subband wave functions. The tilt asymmetrically localizes the subband states on either side of the well.

For the tilted wide well we find that we can ignore $V_{1100}$ and $V_{0011}$ in the regime $0 \leq \gamma \leq 0.7$ and $\gamma=1$ to yield a simple effective model, 


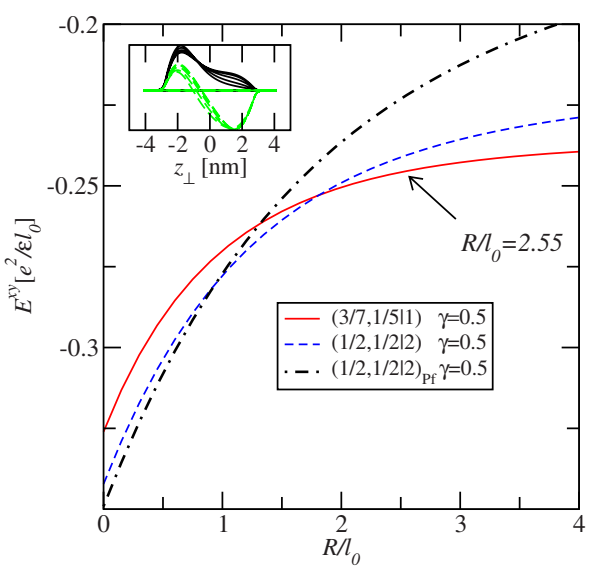

FIG. 2. (Color online) In-plane correlation energies of several states, Eqs. (3) and (4), at $\gamma=0.5$ plotted versus the intersubband interaction parameter $R$. The arrow indicates the parameter relevant for the tilted well samples of Ref. 15 , where the $(3 / 7,1 / 5 \mid 1)$ state is shown to have the lowest correlation energy at $\gamma=0.5$. Inset: normalized lowest (solid) and first (dashed) subband wave functions plotted versus position in the tilted quantum well for several $\gamma$ between 0 and 1 . In the limit $\gamma \rightarrow 0$ the subbands localize to mimic layer states.

$$
H_{\gamma}^{\mathrm{eff}} \approx H_{\gamma}^{\perp}+\frac{1}{2} \sum_{\sigma, \sigma^{\prime},\{m\}} V_{\{m\}}^{\sigma \sigma^{\prime} \sigma \sigma^{\prime}} c_{m_{1} \sigma}^{\dagger} c_{m_{2} \sigma^{\prime}}^{\dagger} c_{m_{4} \sigma^{\prime}} c_{m_{3} \sigma} .
$$

The off-diagonal terms give Haldane pseudopotentials ${ }^{22}$ that are smaller by more than a factor of 7 than the diagonal terms. We assume that diagonal repulsion dominates the intersubband energetics and that the omission of these small off-diagonal terms does not change the energetic ordering of trial wave functions computed below using Eq. (2).

We model the planar interaction computed with the subband wave functions by a modified Zhang-Das Sarma ${ }^{23}$ potential,

$$
V^{\mathrm{MZDS}}=\left(r^{2}+d_{\{\sigma\}}^{2} / l_{0}^{2}\right)^{-1 / 2}+c_{\{\sigma\}}\left(r^{2}+W_{\{\sigma\}}^{2} / l_{0}^{2}\right)^{-1 / 2},
$$

where the parameters $d, c$, and $W$ are determined by fitting lowest pseudopotentials of $V^{\mathrm{MZDS}}$ to those of the interaction computed using the subband wave functions. For nontilted wide wells the $c$ term must be retained. We find good fits with the largest uncertainty $(<5 \%)$ in the $m=1$ pseudopotential. We have checked, by varying fitting parameters, that $5 \%$ variation in pseudopotentials does not qualitatively alter our results.

For the tilted well sample of Ref. 15 we focus on the regime $0 \leq \gamma \leqq 0.7$ and $\gamma=1$ and keep only the $V_{\sigma \sigma^{\prime} \sigma \sigma^{\prime}}$ terms. We then require only three fitting parameters: $d_{0}(\gamma) \equiv d_{0000}$, $d_{1}(\gamma) \equiv d_{1111}$, and $R(\gamma) \equiv d_{1010}$ to approximately match all pseudopotentials. For example, we find the lowest $E^{\text {total }}$ to occur at: $d_{0}(0.5)=1.16 l_{0}, d_{1}(0.5)=1.37 l_{0}$, and $R(0.5)=2.55 l_{0}$.

Equation (2) resembles models of bilayers and very wide wells but there are crucial differences: (i) there is a $\Delta_{\mu}^{\gamma}$ term that favors partial subband polarization, (ii) all terms are functions of the subband polarization, $\gamma$, (iii) the interaction within each subband differs, e.g., $V_{1111} \neq V_{0000}$, and (iv) in
TABLE I. Several possible states, Eq. (3), at half filling. The last column shows that two of the incompressible states are locked at partially polarized configurations.

\begin{tabular}{lccc}
\hline \hline$\left(\bar{\nu}_{0}, \bar{\nu}_{1} \mid m\right)$ & $n_{0}$ & $n_{1}$ & $\gamma$ \\
\hline$(1 / 3,1 / 3 \mid 1)$ & 1 & 1 & 0 \\
$(3 / 7,1 / 5 \mid 1)$ & 3 & 1 & $1 / 2$ \\
$(5 / 11,1 / 7 \mid 1)$ & 5 & 1 & $2 / 3$ \\
$(1 / 2,1 / 2 \mid 2)$ & $\infty$ & $\infty$ & Any \\
$(1 / 4,1 / 4 \mid 0)$ & $\infty$ & $\infty$ & 0 \\
\hline \hline
\end{tabular}

the absence of tilt we must keep off-diagonal terms of the form $V_{\sigma \sigma \sigma^{\prime} \sigma^{\prime}}$.

We now construct competitive two-component variational wave functions expected to minimize the energy of Eq. (2) and therefore $H^{3 \mathrm{D}}$. The single-component LLL CF wave functions at filling $n /(2 p n \pm 1), \psi_{n /(2 p n \pm 1)}$, are given in the literature. ${ }^{3,4}$ The single-component $\mathrm{CF}$ wave functions can be generalized to capture two-component states, ${ }^{18}$

$$
\Psi_{\left(\bar{\nu}_{0}, \bar{\nu}_{1} \mid m\right)}=\prod_{r, j}\left(z_{j}-w_{r}\right)^{m} \psi_{\bar{\nu}_{0}}\left[\left\{z_{k}\right\}\right] \psi_{\bar{\nu}_{1}}\left[\left\{w_{s}\right\}\right],
$$

where the fully antisymmetric wave function $\psi_{\bar{\nu}}$ is a single-component state at filling factor $\bar{\nu}$. Halperin's wave functions ${ }^{7}$ are obtained as special cases for $\bar{\nu}_{0}=1 / m^{\prime}$ and $\bar{\nu}_{1}=1 / \mathrm{m}^{\prime \prime}$. In order to ensure that the electrons of each component occupy the same area, $N_{0}$ and $N_{1}$ must be related by $N_{0} \bar{\nu}_{0}^{-1}+m N_{1}=N_{1} \bar{\nu}_{1}^{-1}+m N_{0}$, thus $\nu=\left({\overline{\nu_{0}}}^{1}+m N_{1} / N_{0}\right)^{-1}+\left(\bar{\nu}_{1}^{1}+m N_{0} / N_{1}\right)^{-1}$. Some of the $\gamma=0$ states of Eq. (3) have been shown to be energetically competitive with favorable ground states at several different filling factors in bilayer systems (small tunneling and equal subband population). ${ }^{18}$ Table I lists a larger set. The constituent wave functions, $\psi_{\bar{\nu}}$, with the lowest energy gap yield an upper bound for the energy gap of each multicomponent state. For example, the gap of the $(3 / 7,1 / 5 \mid 1)$ state is no larger than that of the $\psi_{1 / 5}$ state while $(1 / 2,1 / 2 \mid 2)$ is a gapless CF Fermi sea. ${ }^{4}$ We also include the multicomponent Pfaffian states in our comparison,

$$
\Psi_{\left(\bar{\nu}_{0}, \bar{\nu}_{1} \mid m\right)_{\mathrm{Pf}}}=\prod_{r, j}\left(z_{j}-w_{r}\right)^{m} \psi_{\mathrm{Pf}}\left[\left\{z_{k}\right\}\right] \psi_{\mathrm{Pf}}\left[\left\{w_{s}\right\}\right],
$$

where $\psi_{\text {Pf }}$ is the Pfaffian wave function. ${ }^{9}$

We compute the ground-state energies of several candidate wave functions in the subband basis at $\nu=1 / 2$ for the parameters of Ref. 15 using variational Monte Carlo. We use the spherical geometry ${ }^{4}$ to compute energies in finite-size systems and extrapolate our results to the thermodynamic limit by adding the background energy: $E_{\mathrm{b}}=-\left[N_{1}^{2} F\left(d_{1}\right)+N_{0}^{2} F\left(d_{0}\right)+2 N_{0} N_{1} F(R)\right] / 2$, where $F(x)$ $\equiv\left(e^{2} / 2 \epsilon l_{0}\right)\left(-x / R_{s}+\sqrt{4+x^{2} / R_{s}^{2}}\right)$ and $R_{s}$ is the radius of the sphere in units of the magnetic length.

Figure 2 shows a representative comparison of $E^{x y}$ for three of the lowest energy states at $\gamma=0.5$. Here the parameter $R$ is varied to test the robustness of the lowest energy state. The Monte Carlo error bars are smaller than the linewidth. The lowest energy state at $\gamma=0.5$ is found to be the 
TABLE II. Comparison of planar correlation energies (third column) and confinement energies (fourth column) for several $\nu=1 / 2$ states. The energies were computed for parameters relevant for the tilted well sample of Ref. 15. The lowest total energy is found for the incompressible $(3 / 7,1 / 5 \mid 1)$ state.

\begin{tabular}{lcclc}
\hline \hline$\left(\bar{\nu}_{1}, \bar{\nu}_{2} \mid m\right)$ & $\gamma$ & $E^{x y}\left[e^{2} / \epsilon l_{0}\right]$ & $E^{\perp}\left[e^{2} / \epsilon \bar{l}\right]$ & $E^{\mathrm{total}}\left[e^{2} / \overline{\epsilon l}\right]$ \\
\hline$(1 / 3,1 / 3 \mid 1)$ & 0 & $-0.24858(2)$ & 0.045 & $-0.20358(2)$ \\
$(3 / 7,1 / 5 \mid 1)$ & $1 / 2$ & $-0.24542(4)$ & 0.00036 & $-0.24506(4)$ \\
$(1 / 2,1 / 2 \mid 2)$ & $1 / 2$ & $-0.2440(4)$ & 0.00036 & $-0.2436(4)$ \\
$(1 / 2,1 / 2 \mid 2)_{\mathrm{Pf}}$ & $1 / 2$ & $-0.2214(4)$ & 0.00036 & $-0.2210(4)$ \\
$(1 / 2,0 \mid 0)$ & 1 & $-0.246394(4)$ & 0.0035 & $-0.242894(4)$ \\
$(1 / 2,0 \mid 0)_{\mathrm{Pf}}$ & 1 & $-0.24338(5)$ & 0.0035 & $-0.23988(5)$ \\
\hline \hline
\end{tabular}

incompressible $(3 / 7,1 / 5 \mid 1)$ state. The compressible Fermi sea state, $(1 / 2,1 / 2 \mid 2)$, is nearby in energy. The Pfaffian state becomes lower in energy for the unphysical regime of $R<l_{o}$.

We have compared wave functions at several subband polarizations to globally minimize the total energy. We expect partially subband polarized states to be favored by the competition between the Coulomb interaction along the direction perpendicular to the plane and the quantum-well tilt. We find that the small gain in $E^{\perp}$ favors the $(3 / 7,1 / 5 \mid 1)$ state over all others in a parameter window corresponding to the parameters relevant for the tilted well experiments of Ref. 15, $\alpha=0.3 \mathrm{meV} / \mathrm{nm}$, see Table II. We also conclude that small changes in $\alpha$ will favor the partially subband polarized compressible state, $(1 / 2,1 / 2 \mid 2)$.
Our procedure provides the following physical picture of the experiments in Ref. 15 at $\nu=1 / 2$. A symmetric wide well favors the compressible $(1 / 2,1 / 2 \mid 2)$ state with $\gamma \approx 0$ [or the incompressible $\gamma=0(1 / 3,1 / 3 \mid 1)$ state depending on $\rho$ and $w]$. As the density is made more asymmetric via a tilt the $\mathrm{CF}$ Fermi sea state continuously depopulates its higher subband (it is a subband paramagnet). Near $\alpha \approx 0.3 \mathrm{meV} / \mathrm{nm}$ each subband becomes localized on either side of the well to approximate layerlike behavior but with asymmetric parameters in the subband basis. The incompressible asymmetric state, $(3 / 7,1 / 5 \mid 1)$, is then favored in a narrow parameter window. We find that this state is responsible for the indications of FQHE observed under tilt in Ref. 15. As the state is made more asymmetric the higher subband state becomes further depopulated to again favor $(1 / 2,1 / 2 \mid 2)$.

We have shown that quantum wells with two active subbands can exhibit a wide variety of partially subband polarized, even-denominator FQHE states. Our procedure can be used to find parameter regimes, where incompressible partially subband polarized FQHE states arise as a function of sample density, width, and tilt (e.g., a mixed paired state ${ }^{24}$ ). Equation (3) also provides candidates for recently observed $^{14,15}$ LLL $\nu=1 / 4$ features [e.g., $(3 / 13,1 / 7 \mid 3)$ ], and the second LL FQHE, where tilt can be used to tune between a variety of subband polarizations thereby allowing one to engineer a larger class of interaction potentials. Furthermore, Eq. (3) offers several candidate partially polarized real-spin FQHE states.

We thank S. Das Sarma, J. K. Jain, and M. Shayegan for helpful discussions. M.R.P. was supported by Microsoft Q.
${ }^{1}$ D. C. Tsui, H. L. Störmer, and A. C. Gossard, Phys. Rev. Lett. 48, 1559 (1982); W. Pan, J. S. Xia, H. L. Störmer, D. C. Tsui, C. Vicente, E. D. Adams, N. S. Sullivan, L. N. Pfeiffer, K. W. Baldwin, and K. W. West, Phys. Rev. B 77, 075307 (2008).

${ }^{2}$ R. B. Laughlin, Phys. Rev. Lett. 50, 1395 (1983).

${ }^{3}$ J. K. Jain, Phys. Rev. Lett. 63, 199 (1989).

${ }^{4}$ J. K. Jain, Composite Fermions (Cambridge University Press, Cambridge, England, 2007).

${ }^{5}$ Y. W. Suen, L. W. Engel, M. B. Santos, M. Shayegan, and D. C. Tsui, Phys. Rev. Lett. 68, 1379 (1992); J. P. Eisenstein, G. S. Boebinger, L. N. Pfeiffer, K. W. West, and S. He, ibid. 68, 1383 (1992); Y. W. Suen, M. B. Santos, and M. Shayegan, ibid. 69, 3551 (1992); Y. W. Suen, H. C. Manoharan, X. Ying, M. B. Santos, and M. Shayegan, ibid. 72, 3405 (1994).

${ }^{6}$ R. Willett, J. P. Eisenstein, H. L. Störmer, D. C. Tsui, A. C. Gossard, and J. H. English, Phys. Rev. Lett. 59, 1776 (1987).

${ }^{7}$ B. I. Halperin, Helv. Phys. Acta 56, 75 (1983).

${ }^{8}$ E. H. Rezayi and F. D. M. Haldane, Phys. Rev. Lett. 84, 4685 (2000).

${ }^{9}$ G. Moore and N. Read, Nucl. Phys. B 360, 362 (1991).

${ }^{10} \mathrm{~J}$. Leinaas and J. Myrheim, Nuovo Cimento B 37, Number 1 (1977); F. Wilczek, Phys. Rev. Lett. 48, 1144 (1982).

${ }^{11}$ A. Kitaev, Ann. Phys. 303, 2 (2003); C. Nayak et al., Rev. Mod. Phys. 80, 1083 (2008).

${ }^{12}$ M. Storni, R. H. Morf, and S. Das Sarma, Phys. Rev. Lett. 104,
076803 (2010)

${ }^{13}$ M. R. Peterson, T. Jolicoeur, and S. Das Sarma, Phys. Rev. Lett. 101, 016807 (2008); M. R. Peterson, T. Jolicoeur, and S. Das Sarma, Phys. Rev. B 78, 155308 (2008).

${ }^{14}$ D. R. Luhman, W. Pan, D. C. Tsui, L. N. Pfeiffer, K. W. Baldwin, and K. W. West, Phys. Rev. Lett. 101, 266804 (2008); J. Shabani, T. Gokmen, and M. Shayegan, ibid. 103, 046805 (2009); J. Zhang, R. R. Du, J. A. Simmons, and J. L. Reno, Phys. Rev. B 81, 041308(R) (2010).

${ }^{15}$ J. Shabani, T. Gokmen, Y. T. Chiu, and M. Shayegan, Phys. Rev. Lett. 103, 256802 (2009).

${ }^{16}$ Z. Papic, G. Moller, M. V. Milovanovic, N. Regnault, and M. O. Goerbig, Phys. Rev. B 79, 245325 (2009); Z. Papic, N. Regnault, and S. Das Sarma, ibid. 80, 201303 (2009).

${ }^{17}$ M. R. Peterson and S. Das Sarma, Phys. Rev. B 81, 165304 (2010).

${ }^{18}$ V. W. Scarola and J. K. Jain, Phys. Rev. B 64, 085313 (2001).

${ }^{19}$ M. W. Ortalano, S. He, and S. Das Sarma, Phys. Rev. B 55, 7702 (1997).

${ }^{20}$ L. Hedin and B. I. Lundqvist, J. Phys. C 4, 2064 (1971).

${ }^{21}$ S. H. Vosko et al., Can. J. Phys. 58, 1200 (1980).

${ }^{22}$ F. D. M. Haldane, Phys. Rev. Lett. 51, 605 (1983).

${ }^{23}$ F. C. Zhang and S. Das Sarma, Phys. Rev. B 33, 2903 (1986).

${ }^{24}$ V. W. Scarola, J. K. Jain, and E. H. Rezayi, Phys. Rev. Lett. 88, 216804 (2002). 